\title{
Induced Radioactivity
}

\section{By Dr. C. D. Ellis, F.R.S., Lecturer in Physies, University of Cambridge}

$\mathrm{I}^{\mathrm{T}}$ is just over a year since M. and Mme. CurieJoliot announced that they had succeeded in producing radioactive atoms of low atomic number. In their first experiments they discovered that, when aluminium was bombarded by $\alpha$-particles, a radioactive isotope of phosphorus was produced according to the reaction :

$$
{ }_{13} \mathrm{Al}^{27}+{ }_{2} \mathrm{He}^{4} \rightarrow{ }_{15} \mathrm{P}^{30}+{ }_{0} n^{1} .
$$

The behaviour of this radio-phosphorus was quite analogous to that of the naturally occurring radioactive elements, except that instead of $\beta$-rays, that is negative electrons, positrons were emitted, resulting in the formation of a known stable isotope of silicon:

$$
{ }_{15} \mathrm{P}^{30} \rightarrow{ }_{14} \mathrm{Si}^{30}+\stackrel{+}{\varepsilon} .
$$

The half period of the radioactive decay was about $3 \cdot 2$ minutes.

Similar results were found to occur using boron or magnesium instead of aluminium. With the ordinarily available $\alpha$-particle sources, the amounts of these radioactive bodies that are produced is extremely small. Even if all the $\alpha$-particles emitted from the source are arranged to hit the aluminium, the resulting radioactive phosphorus will have only about one millionth to one tenmillionth of the activity of the source. These small intensities are responsible for the main difficulties in the experiments, which would be impossible but for the delicate counting devices devised by Geiger.

Since the initial discovery of the Joliots, other methods, as suggested in fact by them, of forming radioactive elements have been discovered. Protons, deuterons and neutrons have all been found to lead to nuclear reactions which produce radioactive products. This is not the occasion to give a list of all the new radioactive isotopes that have been discovered. It will suffice to say that more than fifty have already been identified. One important aspect of this may be noted. Aston's work in furnishing a list of all the stable isotopes at least made possible speculations about the structure of nuclei-why certain combinations of protons and neutrons did occur and others did not. His later and more delicate work on the exact masses of the isotopes gave information about the energy of binding of these nuclei and provided a basis for quantitative theories. Now, directly following from the Joliots' discovery, this field is suddenly enriched by a large number of new nuclei about which, by appropriate experiments, we may reasonably hope to obtain similar information. Above all, this discovery must be considered as a remarkable extension of the possibilities of our knowledge.

It is interesting to consider the relation of these new radioactive isotopes to the already known stable isotopes. It is clear that certain combinations of protons and neutrons can form permanent nuclei. The criterion of this permanency lies in the fundamental laws of interaction of proton and neutron, but the question whether a given nucleus is absolutely permanent or radioactive is connected with what might be termed the possibility of existence of neighbouring nuclei. Whether a given nucleus $A$ is stable or radioactive depends, so far as our present knowledge goes, simply on the question whether the reaction $A \rightarrow B+C$ is endothermic or exothermic, $B$ and $C$ being other possible nuclei. In practice, the only reasonable possibilities for $C$ are proton, neutron, $\alpha$-particle, electron or positron. In the natural radioactive elements, $C$ is either an $\alpha$-particle or an electron ; in the newly discovered radioactive elements, $C$ is either an electron or a positron. As yet, no case is known of induced radioactivity with emission of a heavy particle.

In a general way, it is easy to see the factors which determine whether positrons or electrons are emitted. Assuming that nuclei are built up of combinations of protons and neutrons, an examination of the list of the known stable isotopes shows clearly that permanently stable nuclei are only formed when the ratio of the number of neutrons to protons lies within a narrow range. Isotopes with either a smaller or greater neutron/proton ratio do not occur naturally. Now the addition of an $\alpha$-particle (two neutrons + two protons) and the emission of a neutron is, in effect, an addition of one neutron and two protons, and means, therefore, a lowering of the neutron/proton ratio. If this ratio for the new element lies outside the stability range, the element will be radioactive, and the change will clearly be in the direction of raising the neutron/proton ratio. This can be effected by the switch of a proton into a neutron with emission of a positron. On the other hand, if the initial absorption of an $\alpha$-particle causes the emission of a proton, the situation is exactly reversed and a radioactive element so formed will emit negative electrons accompanied by a nuclear switch of a neutron into a proton. As an example of this latter process we may take magnesium bombarded by $\alpha$-particles Magnesium has three stable 
isotopes and one of them undergoes the following reaction :

$$
{ }_{12} \mathrm{Mg}^{25}+{ }_{2} \mathrm{He}^{4} \rightarrow{ }_{13} \mathrm{Al}^{28}+{ }_{1} \mathrm{H}^{1} .
$$

The aluminium isotope so formed is radioactive with a period of two and three quarter minutes, with emission of negative electrons.

The use of protons and deuterons to cause nuclear reactions leading to radioactive products has already been referred to but is of especial interest, since the intensity of the bombarding. beams is here under control, and by sufficient technical application can be largely increased. Already Lawrence has produced a radioactive body in amounts comparable with that of the naturally occurring radioactive substances. A particularly interesting example is that of sodium bombarded by deuterons, when the following reaction occurs :

$$
{ }_{11} \mathrm{Na}^{23}+{ }_{1} \mathrm{D}^{2} \rightarrow{ }_{11} \mathrm{Na}^{24}+{ }_{1} \mathrm{H}^{1} .
$$

This new isotope of sodium disintegrates under emission of $\beta$-particles with a period of fifteen and a half hours. The $\beta$-particles are not particularly energetic, having an upper limit of energy of only about one million volts, but an intense highfrequency $\gamma$-radiation of about five and a half million volts is also emitted. Using 1 microampere of 1.7 million volt deuterons for one hour gave already a two hundredth of a millicurie of radioactive material. This is an amount the effects of which can be detected by ordinary ionisation methods, and there is every reason to believe that it will be possible soon to increase the yield very greatly.

A great number of interesting investigations will be rendered feasible when it is possible to obtain in quantity a radioactive body such as this with a conveniently long period and emitting such high-frequency $\gamma$-radiation.

The most striking results, from the point of view of the number of new radioactive elements produced, have been obtained by Fermi, using neutrons as bombarding particles. The neutrons for these experiments are obtained by allowing $\alpha$-particles to fall on beryllium, in practice by filling a small tube with beryllium and a certain quantity of radon. The number of neutrons is only about one hundred thousandth of the number of $\alpha$-particles emitted by the source, but their efficiency in producing nuclear reactions is much greater. In fact, a large percentage of the neutrons which hit the nucleus produce an active atom. These experiments have also a far greater range than those using $\alpha$-particles or protons, since the absence of charge on the neutron removes any distinction between light and heavy elements.
The new radioactive isotopes formed in this way are spread fairly uniformly throughout the periodic table ; for example, active bodies are formed from fluorine and magnesium, and also from thorium and uranium. In general, for light elements the process of activation consists in the capture of the neutron and simultaneous emission of an $\alpha$ particle or proton. The resulting nucleus has then a higher neutron/proton ratio than corresponds to stability, and the necessary balance is re-established by the radioactive change of a neutron into a proton inside the nucleus with emission of an electron. For elements of higher atomic number, the chance of the initial ejection of a positively charged $\alpha$-particle or proton is diminished by the stronger attractive field of the nucleus, and the initial process is more likely then to be just a simple capture of the neutron, again leading to an active nucleus with too high a neutron/proton ratio.

There are several points about the processes involved in the direct capture of a neutron which are not at present easy to understand, but it is plausible that the probability of capture should increase as the energy of the neutron is decreased. Fermi has in fact shown that the efficiency of production of some radioactive elements may be increased ten to one hundred times by surrounding the source and target by paraffin or water during bombardment. The primary neutrons emitted from the source are slowed down by elastic collisions with the hydrogen nuclei, and in this state are more easily captured by the nuclei of the bombarded substance.

Finally, as an example of the flexibility of these methods of producing new nuclei, we may consider the formation of a certain radioactive isotope of aluminium, ${ }_{13} \mathrm{Al}^{28}$. This can be formed now in no less than five distinct ways, starting from different substances and using different bombarding particles.

Neutrons fired on to either aluminium, silicon, or phosphorus form this body according to the schemes :

$$
\begin{aligned}
& { }_{13} \mathrm{Al}^{27}+{ }_{0} n^{1} \rightarrow{ }_{13} \mathrm{Al}^{28} . \\
& { }_{14} \mathrm{Si}^{28}+{ }_{0} n^{1} \rightarrow{ }_{13} \mathrm{Al}^{28}+{ }_{1} \mathrm{H}^{1} \\
& { }_{15} \mathrm{P}^{31}+{ }_{0} n^{1} \rightarrow{ }_{13} \mathrm{Al}^{28}+{ }_{2} \mathrm{He}^{4} .
\end{aligned}
$$

One can also use deuterons on aluminium or $\alpha$-particles on magnesium, the latter reaction having already been mentioned in another connexion :

$$
\begin{aligned}
& { }_{13} \mathrm{Al}^{23}+{ }_{1} \mathrm{D}^{2} \rightarrow{ }_{13} \mathrm{Al}^{28}+{ }_{1} \mathrm{H}^{1} \\
& { }_{12} \mathrm{Mg}^{25}+{ }_{2} \mathrm{He}^{4} \rightarrow{ }_{13} \mathrm{Al}^{28}+{ }_{1} \mathrm{H}^{1}
\end{aligned}
$$

In every case the same final product is obtained, which emits $\beta$-particles to form ${ }_{14} \mathrm{Si}^{28}$ and has a period of about two and three quarter minutes. 